\title{
AN ADAPTIVE MUUTIGRID ALGORITHM FOR REGION OF INTEREST DIFFUSE OPTICAL TOMOGRAPHY
}

Murat Guven, Birsen Yazici

\author{
Drexel University \\ Electrical and Computer Engineering \\ Philadelphia, PA, USA
}

\author{
Xavier Intes, Britton Chance \\ University of Pennsylvania \\ Department of Biophysics and Biochemistry \\ Philadelphia, PA, USA
}

\begin{abstract}
Due to diffuse nature of light photons, Diffuse Optical Tomography (DOT) image reconstruction is a challenging 3D problem with a relatively large number of unknowns and limited measurements. As a result, the computational complexity of the existing DOT image reconstruction algorithms remains prohibitive.

In this work, we investigate an adaptive multigrid approach to improve the computational efficiency and the quantitative accuracy of DOT image reconstruction. The key idea is based on locally refined grid structure for region of interest (ROI). The ROI may be defined as diagnostically significant regions, strong background heterogeneities and/or deep optical edges. A 2-level mesh is generated to provide high resolution for ROI and sufficiently high resolution for the rest of the image. A Least Squares (LS) solution is formulated for the inverse problem. Fast Adaptive Composite (FAC) 2-Grid algorithm is employed to solve the inverse problem. Conjugate Gradient (CG) is used at the relaxation stage of FAC 2-Grid. Same problem is also solved using direct CG and standard 2-Grid method for globally fine grid structure. Our numerical studies demonstrate that the proposed FAC based adaptive 2-Grid approach provides up to $90 \%$ reduction in computational requirements as compared to the direct iterative and standard 2-Grid methods while providing better image quality. The fundamental ideas introduced in this study are directly applicable to other linear and nonlinear inverse problems with Newton type global linearization.
\end{abstract}

\section{INTRODUCTION}

Diffuse Optical Tomography (DOT) is a non-invasive imaging modality that uses near infrared (NIR) light to reveal spatial distribution of the optical properties of deep biological tissue. It offers unique information about the anatomy, physiology, biochemistry and molecular functions of soft tissue that is not available from the existing imaging modalities [5]. Most important current applications of this technology are in breast cancer detection, cognitive activity monitoring, and brain tumor and hemorthage detection 15 8].

The propagation of light in the tissue is not restricted to a plane. This makes the optical image reconstruction a challenging 3D inverse problem with a relatively large number of unknowns and limited measurements. Currently, high spatial resolution of magnetic resonance imaging (MRl) or X-ray computed tomography (CT) is beyond the capability of NIR tomography due to diffuse nature of light and prohibitive computational requirements. In this study, we propose to balance the two opposing issues resolution/accuracy versus computational complexity within an adaptive multigrid framework.

To motivate the adaptive multigrid methodology, we briefly summarize the standard multigrid problem solving methodology. Multigrid solvers have the unique potential of solving many kinds of mathematical problems with $\mathrm{N}$ unknowns with $O(N)$ work load (Note that direct iterative techniques theoretically converge in $\left.O\left(N^{2}\right)\right)$. This includes integral equations, optimization methods in various scientific disciplines and the solution of partial differential equations. Recently, multigrid algorithms have been applied to a variety of problems arising in control theory, combinatorial optimization, statistical mechanics, with a growing list of problems in image processing. Standard multigrid uses a hierarchy of coarser and coarser grids to accelerate the convergence of standard iterative methods. The basic idea in multigrid algorithms consists of approximating the smooth error by restricting to a coarser grid on which it appears again as a high frequency error and is therefore easily eliminated by the iterative solver on the coarse grid. Standard multigrid methods have been applied to DOT image reconstruction problem recently [9], where nonlinear multigrid methods were applied to solve fully nonlinear problem via Newton type linearization process employed at every grid level.

In most medical imaging applications, geometric prior information regarding the unknown optical image is available either from a secondary imaging modality or from human anatomy. Therefore, one can prespecify a ROI that contains diagnostically pertinent information during the process of image reconstruction. This ROI may require substantially enhanced high spatial resolution which may be well beyond the requirements of the rest of the image. In this case, it is wasteful to let ROI requirements dictate the global parameters of the image reconstruction process. Adaptive multigrid methods allow us to effectively treat the requirements of the ROI and dynamically refine the image reconstruction during the solution process. Note that one may consider to partition the image volume into nonuniform voxels and utilize direct iterative techniques to reconstruct an image with spatially varying resolution. However, multigrid, in particular adaptive multigrid methods are fast iterative solvers developed to reduce the computational requirements of the direct iterative solver while improving the achievable quantitative accuracy.

In this work, we focus to improve the computational efficiency and the quantitative accuracy of DOT by formulating a Fast Adaptive Composite (FAC) 2-Grid algorithm $[1,10]$. An adaptively refined 2-level composite grid, which is based on the ROI information and corresponding spatial resolution requirements, is gener- 
ated to provide higher resolution for ROI and sufficiently high resolution for the background (BG). A least squares solution is formulated for the $2 \mathrm{D}$ inverse problem and a conjugate gradient method is employed at the relaxation stage of the multigrid solver. The reconstruction results are presented to compare the quantitative accuracy and computational advantages of the proposed algorithm over standard least squares and standard 2-Grid algorithms.

\section{THEORY}

\subsection{Forward Model}

Photon propagation in soft tissue can be adequately modeled by the diffusion equation. In this work, we focus on the reconstruction of absorption coefficients, hence we keep the scattering coefficient and consequently the diffusion coefficient constant throughout our calculations. As a result, we make use of the following frequency domain diffusion equation as a forward model:

$$
-\frac{i \omega}{c} \phi(r)-D \nabla^{2} \phi(r)+\mu_{a}(r) \phi(r)=A \delta\left(r_{s}\right)
$$

$\phi(r)$ represents the spatially varying total field due to the point source $A \delta\left(r_{s}\right)$ located at $r=r_{s} . \omega$ denotes the frequency, $c$ is the speed of light and $i=\sqrt{-1} . D$ is the spatially invariant diffusion coefficient and $\mu_{a}(r)$ stands for the spatially varying absorption coefficient.

The nonlinear relationship that is observed between $\mu_{a}(r)$ and $\phi(r)$ in Eq. (1) increases the computational burden in the reconstruction of the absorption coefficient distribution. In order to overcome this difficulty, we use a linearized model.

Let $\phi(r)=f\left(\mu_{a}(r)\right)$ be the nonlinear function relating the spatial distribution of absorption coefficient $\mu_{a}(r)$, to the total field $\phi(r)$. A linear relationship between $\phi(r)$ and $\mu_{a}(r)$ can be obtained using the perturbation theory $[2,3,4]$. We have applied Rytov approach [2] using a first order approximation to express the forward problem.

In the case of DOT, multiple source-detector pairs with multiple frequencies are used. The medium is discretized into $\mathrm{N}$ voxels and the forward problem is expressed in terms of a system of linear equations that relates the diffuse Rytov phase $\Phi_{s c}(r)$ to the spatially varying component $\delta \mu_{a}(r)$ of the absorption coefficient distribution $\mu_{a}(r)$ :

$$
\left[\begin{array}{c}
\Phi_{s c}^{f_{1}}\left(r_{d_{1}}, r_{s_{1}}\right) \\
\vdots \\
\Phi_{s c}^{f_{1}}\left(r_{d_{m}}, r_{s_{m}}\right) \\
\Phi_{s c}^{f_{p}}\left(r_{d_{1}}, r_{s_{1}}\right) \\
\vdots \\
\Phi_{s c}^{f_{p}}\left(r_{d_{m}}, r_{s_{m}}\right)
\end{array}\right]=\left[\begin{array}{ccc}
W_{11}^{f_{1}} & \cdots & W_{1 N}^{f_{1}} \\
\vdots & \ddots & \vdots \\
W_{m 1}^{f_{1}} & \cdots & W_{m N}^{f_{1}} \\
W_{11}^{f_{p}} & \cdots & W_{1 N}^{f_{p}} \\
\vdots & \ddots & \vdots \\
W_{m 1}^{f_{p}} & \cdots & W_{m N}^{f_{p}}
\end{array}\right] \times\left[\begin{array}{c}
\delta \mu_{a}\left(r_{1}\right) \\
\vdots \\
\delta \mu_{a}\left(r_{k}\right) \\
\delta \mu_{a}\left(r_{k+1}\right) \\
\vdots \\
\delta \mu_{a}\left(r_{N}\right)
\end{array}\right]
$$

where $\Phi_{s c}\left(r_{d_{i}}, r_{s_{i}}\right)$ is the real part of the diffuse perturbative Rytov phase for the $i^{\text {th }}$ source-detector pair; $W_{i j}^{f_{k}}$ is the real value of the weight for the $j^{\text {th }}$ pixel and the $i^{\text {th }}$ source-detector pair at frequency $f_{k}$ and $\delta \mu_{a}\left(r_{j}\right)$ is the differential absorption coefficient of the $j^{t h}$ pixel. The relation above can be expressed equivalently as follows:

$$
y=W \times x
$$

where $y$ is the measurement vector holding the perturbative Rytov phase $\Phi_{s c}\left(r, r_{s}\right)$ for each source-detector pair and $W$ denotes the linear forward model (weight matrix) which relates the differential absorption coefficient distribution

$$
x=\left[\begin{array}{llll}
\delta \mu_{a}\left(r_{1}\right) & \delta \mu_{a}\left(r_{2}\right) & \cdots & \delta \mu_{a}\left(r_{N}\right)
\end{array}\right]^{T}
$$

to the measurement vector $y$.

\subsection{Inverse Problem}

In this work we formulated a least squares (LS) solution to solve the inverse problem:

$$
\hat{x}_{L S}=\arg \min _{x}\|y-W x\|_{2}^{2}
$$

where $\hat{x}_{L S}$ is the estimate to the unknown image $x$ and $\|.\|_{2}$ denotes the $L_{2}-$ norm. The motivation for this simple formulation is to highlight the difference between various iterative solutions. The approach can be directly extended to the least square formulation with zero order Tikhonov regularization.

Adaptive multigrid differs from standard multigrid in the sense that a hierarchy of global and local grids is considered from the coarsest level to the finest level (Fig. 1), which eventually reduces the dimension of the inverse problem. We have generated a 2level adaptive composite grid for the unknown image $x$ based on prespecified ROI localization information and the corresponding resolution requirements. The image domain is discretized so that the ROl has the desired fine resolution while the rest of the domain has relatively lower but sufficiently high resolution level. A

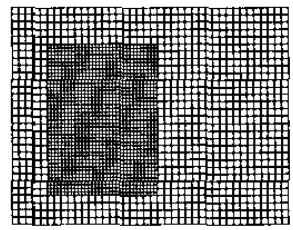

Fig. 1. The composite grid $\Omega^{c}$ designed for the inverse problem solution

Fast Adaptive Composite (FAC) 2-grid algorithm is employed to solve the resulting problem. A hierarchy of grids $\Omega^{2 h}$ and $\Omega_{R O I}^{h}$ is considered where $\Omega^{2 h}$ is the global coarse grid on the whole image with grid size $2 h$ and $\Omega_{R O I}^{h}$ is the local fine grid on the ROl, which is a subset of the global fine grid $\Omega^{h}$, with grid size $h$, combination of which leads to the 2-level composite grid $\Omega^{c}$ (Fig. 1), which in turn provides the required resolution levels for both ROI and BG images.

The algorithm iteratively updates the ROI image estimate on the local fine grid $\Omega_{R O I}^{h}$. The coarse grid problem is solved on the global coarse grid $\Omega^{2 h}$ for the error according to the coarse grid residual equation. The LS formulation on the fine grid $\Omega^{h}$ can be equivalently expressed for the error on the coarse grid $e^{2 h}$ as

$$
\begin{aligned}
\left\|y-W^{h} x^{h}\right\|_{2}^{2} & =\left\|y-W^{h}\left(\hat{x}^{h}+e^{h}\right)\right\|_{2}^{2} \\
& =\left\|y-W^{h}\left(\hat{x}^{h}+I_{2 h}^{h} e^{2 h}\right)\right\|_{2}^{2} \\
& \left.=\| y-W^{h} \hat{x}^{h}-W^{h} I_{2 h}^{h} e^{2 h}\right) \|_{2}^{2} \\
& =\left\|r-W^{2 h} e^{2 h}\right\|_{2}^{2}
\end{aligned}
$$


where the residual $r=y-W^{h} \hat{x}^{h}$ and $W^{2 h}=W^{h} I_{2 h}^{h}$ and $I_{2 h}^{h}$ is the interpolation matrix. We have selected $I_{2 h}^{h}=4\left(I_{h}^{2 h}\right)^{T}$ where $I_{h}^{2 h}$ is the full weighting operator in $2 \mathrm{D}[10]$. lows:

We have formulated the FAC 2-grid algorithm for DOT as fol-

- Split the measurement vector $y$ into its ROI and BG components such that

$$
y=y_{R O I}+y_{B G}
$$

- Initialize the ROI and BG image vectors to zero. Initialize the BG residuals in the coarse level to the BG measurements $y_{B G}$, since

$$
r_{\mathrm{BG}}=y_{\mathrm{BG}}-W_{\mathrm{BG}}^{h} \hat{x}_{B G}^{h}
$$

, where $W_{B G}^{(h)}$ is the weight matrix on $\Omega^{h}$ corresponding to BG image $x_{B G}^{h}$ and $\hat{x}_{B G}^{h}$ is the corresponding image estimate.

\section{2-grid cycle begins}

- Iterate $\nu_{1}$ times on the local fine grid image $x_{R O I}^{h}$ to minimize

$$
\left\|y_{R O I}-W_{R O I}^{h} x_{R O I}^{h}\right\|_{2}^{2}
$$

using CG algorithm.

- Compute the fine-grid residual

$$
r_{R O I}=y_{R O I}-W_{R O I}^{h} \hat{x}_{R O I}^{h}
$$

- Iterate $\nu_{2}$ times on the coarse level ROI error, $e_{R O I}^{2 h}$, with the initial $e_{\mathrm{RO}, 0}^{2 h}$ equal to zero, that is minimize

$$
\left\|r_{R O I}-W_{R O I}^{2 h} e_{R O I}^{2 h}\right\|_{2}^{2}
$$

where $W_{R O I}^{2 h}$ is the weight matrix corresponding to ROI image on the global coarse grid $\Omega^{2 h}$.

- Iterate $\nu_{2}$ times on the coarse grid error $e^{2 h}$, with the initial BG error estimate $\hat{e}_{B G, 0}^{2 h}$ equal to zero, minimizing

$$
\left\|r_{B G}-W_{B G}^{2 h} e_{B G}^{2 h}\right\|_{2}^{2}
$$

- Update the BG image on the global coarse grid $\Omega^{2 h}$.

$$
\hat{x}_{B G}^{2 h}=\hat{x}_{B G}^{2 h}+\hat{e}_{B G}^{2 h}
$$

- Update the residuals for BG image

$$
r_{B G}=y_{B G}-W_{B G}^{2 h} \hat{x}_{B G}^{2 h}
$$

- Interpolate the ROI error $e_{R O I}^{2 h}$ on the global coarse grid to the fine grid level and update the fine grid image estimate $\hat{x}_{R O I}^{h}$.

$$
\begin{gathered}
\hat{e}_{R O I}^{h}=I_{2 h}^{h} \hat{e}_{R O I}^{2 h} \\
\hat{x}_{R O I}^{h}=\hat{x}_{R O I}^{h}+\hat{e}_{R O I}^{h}
\end{gathered}
$$

\section{End of 2-grid cycle}

- Repeat 2-Grid cycles until no significant improvement on the overall image is achieved.

\subsection{Complexity Analysis}

For one iteration of CG algorithm, approximately $7 N P$ number of multiplications is required, where $N$ is the number of pixels of the image on the global fine grid $\Omega^{h}$ and $P$ is the number of measurements. For the standard 2-grid algorithm, the number of the total multiplications becomes $7 N P \nu_{1}+\frac{7 N P}{4} \nu_{2}$, where $\nu_{1}$ is the number of iterations on the global fine grid $\Omega^{h}$ and $\nu_{2}$ is the number of iterations on the global coarse grid $\Omega^{2 h}$.

Making use of composite grids as explained in the previous section reduces the number of multiplications in FAC 2-grid algorithm to $7 N_{R O I} P \nu_{1}+\frac{7 N P}{4} \nu_{2}$, where $N_{R O I}$ is the number of pixels and $\nu_{1}$ is the number of iterations on the local fine grid $\Omega_{R O I}^{h}$ and $\nu_{2}$ is the number of iterations on the global coarse grid $\Omega^{2 h}$.

\section{SIMULATION EXPERIMENTS}

We have simulated optical data for the medium shown in Fig. 5. The circular inclusion on the left has a differential absorption coefficient $\delta \mu_{a}$ of $0.35 \mathrm{~cm}^{-1}$ and the square inclusion on the right has $\delta \mu_{a}$ of $0.2 \mathrm{~cm}^{-1}$. The global fine grid $\Omega^{h}$ is of size $60 \times 80$.

We evaluate the performance of ROI image reconstructions in terms of convergence rate and error norm given as

$$
\epsilon(n)=\left\|x_{R O I}^{h}-\hat{x}_{R O I}^{h,(n)}\right\|_{2}
$$

where $x_{R O I}^{h}$ is the actual ROI image on $\Omega_{R O I}^{h}$, and $\hat{x}_{R O I}^{h,(n)}$ is the ROI image estimate after $n^{\text {th }}$ iteration. Three different algorithms

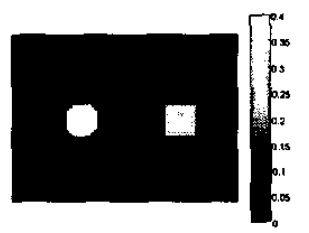

Fig. 2. The original image used to generate synthetic optical measurement data.

are applied for the solution of the inverse problem.

Case 1: For this case, an LS solution is formulated for the inverse problem and a solution is obtained on the fixed grid $\Omega^{h}$ iteratively using a CG algorithm. Fig. 3 . shows the image reconstruction after 1200 iterations and the convergence rate of the solution.
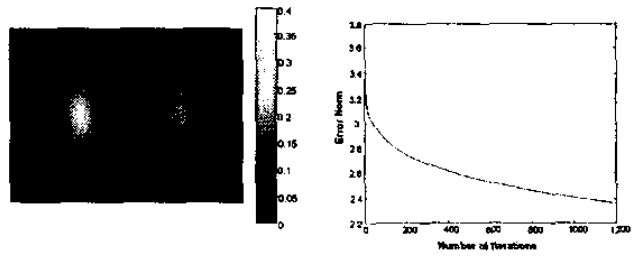

Fig. 3. The image reconstruction and the corresponding error norm vs. iteration number plot for the fixed grid method. 
Table 1. The number of multiplications required to achieve the error norm specified in the first row is tabulated for each algorithm:

\begin{tabular}{|c|c|c|}
\hline 2.6 & 2.2 & 1.65 \\
\hline \hline $18.5 \times 10^{9}$ & Not achieved & Not achieved \\
$13 \times 10^{9}$ & $3 \times 10^{10}$ & Not achieved \\
$1 \times 10^{9}$ & $4 \times 10^{9}$ & $1.7 \times 10^{10}$ \\
\hline
\end{tabular}

Case 2: In this case, the problem is formulated on both $\Omega^{2 h}$ and $\Omega^{h}$, where there is no grid refinement at the fine resolution level $\Omega^{h}$ and a standard 2-grid algorithm is applied. Fig. 4 shows that the rate of convergence accelerates as relaxation procedure passes from one level to the other.
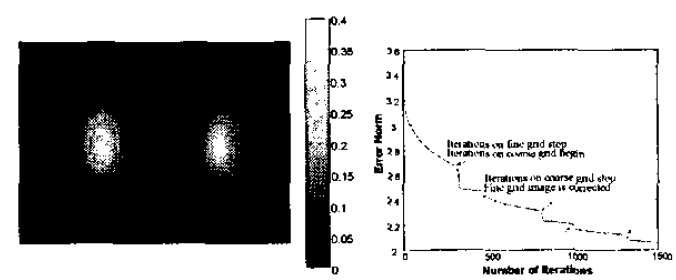

Fig. 4. The image reconstruction and the corresponding error norm vs. iteration number plot for the standard 2-Grid method after 32 grid cycles where $\nu_{1}=300$ and $\nu_{2}=200$. Arrows show the iteration number at which inter-grid operations take place.

Case 3: For the last case the FAC 2-Grid algorithm is applied. Fig. 5 shows the significant enhancement in the image quality especially on ROI. The corresponding error norm vs. iteration number plot approves the improvement in both image quality and computation efficiency relative to the results obtained by fixed grid and standard 2-Grid methods.

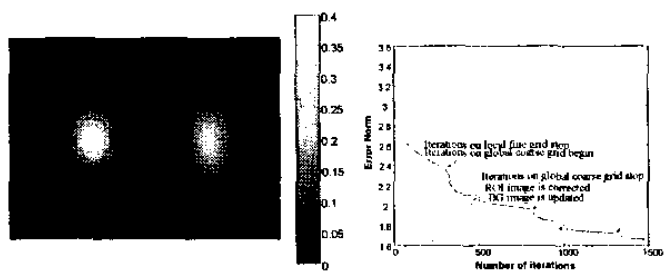

Fig. 5. The image reconstruction and the corresponding error norm vs. iteration number plot for the proposed FAC 2-Grid algorithm after 32 -grid cycles where $\nu_{1}=300$ and $\nu_{2}=200$. $N_{R O I}=1344$. Arrows show the iteration number at which intergrid operations take place.

Finally in Fig. 6, the error-norm vs. multiplication number plots are presented for all algorithms. The plot clearly shows that the computational efficiency is greatly improved by the proposed FAC 2-Grid algorithm. Furthermore the proposed algorithm reduces the error norm to significantly low values. Note that the other two methods can not achieve the same accuracy even if the number of iterations is increased.

\section{CONCLUSION}

The proposed FAC based adaptive 2-Grid approach provides significant reduction in computational requirements as compared to the direct iterative and standard 2-Grid methods while providing better image quality. The fundamental ideas introduced in this study can be easily extended to formulate FAC Multigrid algorithms with regularized least squares formulation, which motivates our future work.

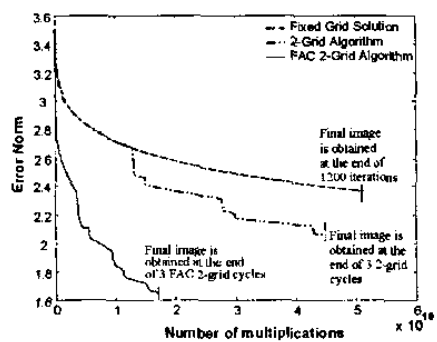

Fig. 6. Error norm vs. multiplication number plots for Case 1,2 and the proposed FAC 2-Grid algorithm.

\section{REFERENCES}

[1] U. Trottenberg, C. Oosterlee, A. Schüller, "Multigrid," Academic Press, New York, 2001

[2] M. A. O'Leary, D. A. Boas, B. Chance, and A. G. Yodh, "Experimental images of heterogeneous turbid media by frequency-domain diffusing-photon tomography," Opt. Lett. 20, pp.426-428, 1995.

[3] S. R. Arridge, "Photon Measurement Density Functions. Part 1: Analytical Forms," Appl. Opt. 34, pp. 7395-7409, 1995.

[4] A. C. Kak, and M. Slaney,"Principles of Computerized Tomographic Imaging," pp. 208-218, IEEE, New York, 1988.

[5] B. Chance, "Optical Methods," Annual Review of Biophysics and Biophysical Chemistry, vol. 20, pp. 1-28, 1991.

[6] J.C. Hebden, S.R. Arridge and D.T. Delpy, "Optical imaging in medicine: I. Experimental techniques," Phys. Med. Biol. Vol. 42, pp. 825-840, 1997.

[7] S. R. Arridge and J. C. Hebden, "Optical imaging in medicine: II. Modeling and reconstruction," Phys. Med. Biol. Vol. 42, pp. 841-853, 1997.

[8] S. Nioka, Q. Luo, and B. Chance, "Human brain functional imaging with reflectance cws," Adv. Exp. Med. Biol., vol. 428, pp. 237.242, 1997.

[9] J. C. Ye, C. A. Bouman, K. J. Webb, and R. Millane, "Nonlinear multigrid algorithms for Bayesian optical diffusion tomography," IEEE Transactions in Image Processing. Vol. 10, No. 6, pp. 909-922, June 2001.

[10] W. L. Briggs, V. E. Henson, S. F. McCormick, "A Multigrid Tutorial," SIAM, Philadelphia, 2000. 